\title{
昡pubvet
}

https://doi.org/10.31533/pubvet.v15n05a812.1-6

\section{Megaesôfago congênito em cão}

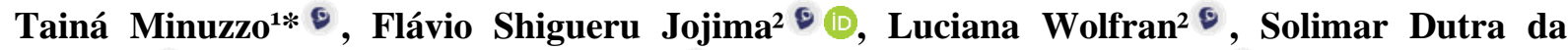 \\ Silveira $^{1}{ }^{\circ}$, Camila Francisca Batschke ${ }^{1} \odot$ (D) Fernanda de Lima Correa $^{1}{ }^{\circ}$, Leticia Maria de \\ Almeida $\operatorname{Santos}^{30}$
}

${ }^{I}$ Médico(a) Veterinário(a) Residente da área de Clínica Médica de Pequenos Animais, Universidade Federal do Paraná, Palotina-PR ${ }^{2}$ Professor adjunto do Departamento de Ciências Veterinárias da Universidade Federal do Paraná - Setor Palotina. ${ }^{3}$ Médica Veterinária Residente da área de Diagnóstico por Imagem, Universidade Federal do Paraná, Palotina-PR. *Autor para correspondência: minuzzotaina@gmail.com

Resumo. O megaesôfago se caracteriza pela hipomotilidade esofágica associada a distensão total ou parcial do órgão, podendo ser classificado como congênito, adquirido idiopático ou adquirido secundário. Sabe-se que a patogenia da forma congênita não é bem elucidada, contudo, os principais sinais clínicos são relatados após o desmame e cursam com quadros de regurgitação, retardo no desenvolvimento do filhote e nos casos mais graves, complicações como pneumonia por aspiração. O diagnóstico é baseado através da anamnese, exame clínico, radiografias (simples e contrastadas) e exclusão de causas secundárias. Já o tratamento, fundamenta-se no manejo alimentar e correção de complicações secundárias. O objetivo deste trabalho é relatar o caso incomum de um cão da raça pinscher, de cinco meses de idade, com queixa de apatia, apetite voraz, regurgitação iniciada após o desmame e perda de peso progressiva. Frente as alterações, foram realizadas radiografias simples e contrastadas, que evidenciaram megaesôfago e após a exclusão de causas secundárias, confirmou-se o diagnóstico de megaesôfago congênito. Realizou-se então, o manejo alimentar, a fim de manter o aporte calórico, com dieta pastosa, fracionada cinco vezes ao dia, com a paciente sendo mantida em posição bipedal durante e após a alimentação. A paciente apresentou melhora significativa do escore corporal e redução dos quadros de regurgitação.

Palavras-chave: Dilatação esofágica, filhote, pinscher, regurgitação.

\section{Congenital megaesophagus in a dog}

Abstract. Megaesophagus is characterized by esophageal hypomotility associated with total or partial organ distension, which can be classified as congenital, acquired idiopathic, or acquired secondary. The pathogenesis of the congenital form is not well elucidated. However, the main clinical signs are reported after weaning with regurgitation, a delay in the puppy development and in the most severe cases, complications as aspiration pneumonia. The diagnosis is based on anamnesis, clinical examination, radiographs (simple and contrasted) and exclusion of secondary causes. Treatmentis based on nutrition management and resolution of secondary complications. Tthis paper aims to report the unusual case of a five-month-old pinscher dog, with signs of apathy, voracious appetite, regurgitation initiated after weaning and progressive weight loss. Due to the alterations simple and contrasted radiographs were performed, which showed megaesophagus. After excluding secondary causes, the diagnosis of congenital megaesophagus was confirmed. Therefore, food management was carried out to maintain caloric intake, with a pasty diet, five times a day, with the patient kept in a bipedal position during and after feeding. The patient showed a significant improvement in body score and reduced regurgitation.

Keywords: Esophageal dilation, puppy, pinscher, regurgitation 


\section{Megaesófago congénito en un perro}

Resumen. El megaesófago se caracteriza por hipomotilidad esofágica asociada con distensión orgánica total o parcial, y puede clasificarse como congénito adquirido idiopático o adquirido secundario. Se sabe que la patogenia de la forma congénita aún no está bien dilucidada, sin embargo, los principales signos clínicos se reportan después del destete y se presentan con regurgitación, retraso en el desarrollo del cachorro y en los casos más severos, complicaciones como la neumonía por aspiración. El diagnóstico se basa en anamnesis, exploración clínica, radiografías (simples y contrastadas) y exclusión de causas secundarias. El tratamiento, por otro lado, se basa en el manejo de los alimentos y la corrección de complicaciones secundarias. El objetivo de este trabajo es reportar el caso inusual de un perro pinscher de cinco meses que se queja de apatía, apetito voraz, regurgitación iniciada después del destete y pérdida de peso progresiva. Ante los cambios se realizaron radiografías simples y contrastadas, las cuales mostraron megaesófago, luego de la exclusión de causas secundarias se confirmó el diagnóstico de megaesófago congénito. Posteriormente, se llevó a cabo un manejo alimentario para mantener el aporte calórico, con una dieta pastosa, dividida cinco veces al día, manteniéndose el paciente en posición bípeda durante y después de la alimentación. El paciente mostró una mejora significativa en la puntuación corporal y una regurgitación reducida.

Palabras clave: Dilatación esofágica, perro, pinscher, regurgitación

\section{Introdução}

O megaesôfago se caracteriza por uma alteração na fisiologia do trânsito alimentar em decorrência da disfunção de motilidade e peristaltismo proveniente de causas como estenose secundária à inflamação da musculatura esofágica, obstruções físicas parciais, desordem de inervação ou persistência do arco aórtico direito, resultando no acúmulo de água, alimento e ar intraesofágico e consequente dilatação passiva do órgão (Gelberg, 2012; Torres, 1997).

O agravamento do quadro pode ser observado quando a estase alimentar leva a fermentação dos nutrientes ali presentes, gerando um ciclo vicioso de isquemia e processo inflamatório crônico da parede esofágica. Inicia-se então, um processo de desnervação progressiva do órgão em decorrência do comprometimento dos plexos mioentéricos e submucoso, que levam a diminuição da amplitude das contrações, assim como, resposta motora mínima ou ausente ao estímulo de deglutição do bolo alimentar, tendendo a paralisia completa do órgão (Strombeck \& Troya, 1976; Torres, 1997).

Pode-se classificar o megaesôfago em congênito, adquirido idiopático ou adquirido secundário (Torres, 1997). Há descrições de predisposição hereditária em algumas raças para o desenvolvimento da afecção, como Fox Terrier, Schnauzer, Pastor Alemão, Dogue Alemão, Golden Retriever e Setter Irlandês (Andrade et al., 2007; Diamant et al., 1974; Mace et al., 2012). Já a espécie felina é pouco acometida, provavelmente devido ao maior predomínio de fibras musculares lisas na sua composição esofágica, apesar disso, a raça com maior propensão é a Siamesa (Sturgess et al., 2001).

Exames complementares que auxiliam na elucidação da afecção incluem a radiografia simples e esofagograma contrastado, que poderá indicar algumas complicações da doença esofágica assim como, dilatação do esôfago. Além disso, cita-se a possibilidade de realização de endoscopia a fim de diagnosticar comorbidades como a presença de parasitas, corpos estranhos, perfurações, neoformações, alimentos, intussuscepção gastroesofágica e redundância da mucosa do esôfago, sugerindo um quadro de megaesôfago (Alves et al., 2013; Tanaka et al., 2010; Torres, 1997).

$\mathrm{O}$ presente relato tem como objetivo descrever um caso incomum de megaesôfago em uma canina de pequeno porte, da raça pinscher, de cinco meses de idade, evidenciando seus aspectos clínicos, método de diagnóstico e terapêutica instituída para o caso.

\section{Material e métodos}

Foi atendido no Hospital Veterinário da Universidade Federal do Paraná - Setor Palotina, no setor de clínica médica de pequenos animais, uma paciente da espécie canina, da raça Pinscher, com cinco 
meses de idade, pesando $2 \mathrm{~kg}$. Durante a anamnese, foi relatado que a paciente apresentava apetite voraz, regurgitação há três meses, fato que coincidiu com o período de desmame e desde então, demonstrando perda de peso progressiva. Ao exame físico foi observado que a paciente se encontrava abaixo do peso (Escore de Condição Corporal 2/9) e apática, com os demais parâmetros fisiológicos dentro da normalidade. Diante das alterações relatadas, foi realizado exames complementares para melhor elucidação do quadro, incluindo hemograma, onde não evidenciou nenhuma alteração, bioquímicos (creatinina, uréia, potássio, sódio, creatina quinase, alanina aminotrasferase, fosfatase alcalina, albumina, colesterol e triglicerídeos) e urinálise, ambos dentro dos parâmetros de normalidade para a espécie. Como avaliação complementar, procedeu-se exame de radiografia simples e contrastada, o qual demonstrou a presença de megaesôfago cervicotorácico, sem complicações secundárias a enfermidade (Figura 1).

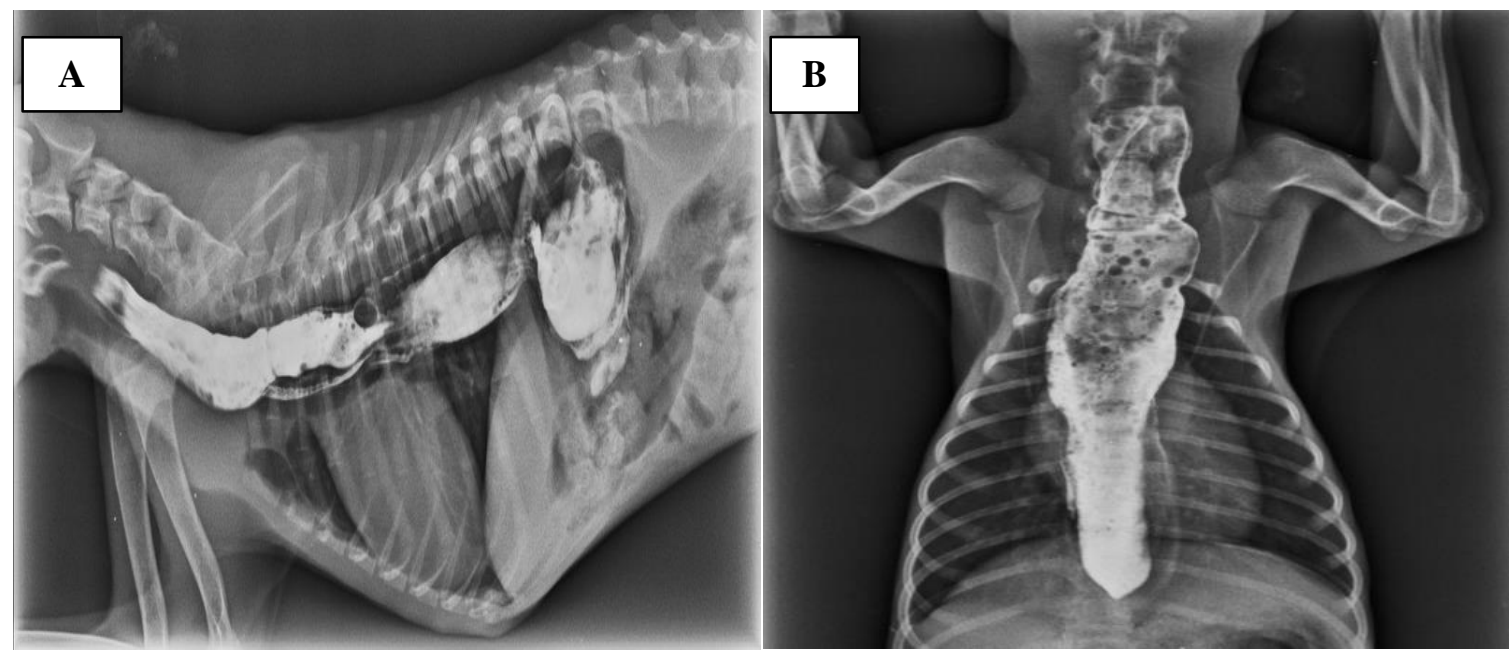

Figura 1. Imagem radiográfica contrastada da região cervicotorácica em incidência lateral esquerda (A) e ventrodorsal (B), de uma canina da raça pinscher de cinco meses de idade. Nota-se presença de dilatação esofágica associada a acúmulo do meio de contraste desde a porção cervical do esôfago até a cárdia, além de deslocamento ventral da traqueia intratorácica e moderada quantidade de conteúdo gasoso (aerofagia) em topografia gástrica. (Fonte: LDI-UFPR)

Devido os resultados obtidos nos exames, foi realizado o cálculo de necessidade energética de manutenção (NEM) com fator de correção de 1,6 estimando que a paciente deveria ingerir $256 \mathrm{kcal} / \mathrm{dia}$, assim sendo, instituiu-se tratamento paliativo através do manejo alimentar, indicando-se o fornecimento de dieta pastosa, fracionada em cinco vezes ao dia, com porções contendo $51 \mathrm{kcal}$ bem como, manter o paciente em posição elevada durante a alimentação e ingestão hídrica, respeitando um ângulo de $45^{\circ} \mathrm{a}$ $90^{\circ}$ ao solo e permanecendo deste mesmo modo, 10 a 15 minutos após o término.

A paciente apresentou uma melhora significativa no escore corporal com redução do quadro de regurgitação após quarenta dias de tratamento, no entanto, veio a óbito em decorrência de um atropelamento.

\section{Resultados e discussão}

Segundo Alves et al. (2013), o megaesôfago congênito refere-se à hipomotilidade e à dilatação do esôfago, provocando quadros de regurgitação, que acarretam perda de peso progressiva e retardo no desenvolvimento do filhote após o desmame. Os sinais clínicos comumente encontrados, além dos já descritos, incluem sialorréia, odinofagia, disfagia e apetite voraz (Tanaka et al., 2010). Tal afirmação corrobora com o presente caso relatado, visto que o animal iniciou os episódios de regurgitação logo após o desmame, da mesma maneira que apresentou apetite voraz, disfagia e consequentemente perda de peso progressiva (Escore de Condição Corporal 2/9).

A paciente acompanhada não apresentou sinais clínicos compatíveis com possíveis complicações secundárias ao megaesôfago, além de não ter apresentado alterações radiográficas sugestivas de pneumonia aspirativa ou outras comorbidades diagnosticadas pelo exame. As alterações respiratórias são normalmente descritas em pacientes com megaesôfago, muitas vezes indicando um quadro de pneumonia por aspiração evidenciados por tosse, taquipneia, pirexia e cianose (Tanaka et al., 2010). 
De acordo com Andrade et al. (2007), o megaesôfago trata-se de uma enfermidade comumente diagnosticada em cães de médio e grande porte, sendo extremamente incomum em raças pequenas Contrariando tal afirmação, pode-se verificar a presença dessa afecção em uma cadela de pequeno porte da raça pinscher, ressaltando a importância da investigação clínica da enfermidade em pacientes não predispostos.

Kook (2013) afirma que se faz necessário a exclusão de causas adquiridas de megaesôfago para se comprovar a forma congênita idiopática. Assim sendo, a miastenia gravis é uma afecção primária comumente relacionada ao megaesôfago adquirido, acometendo principalmente cães na faixa etária de dois a quatro anos de idade ou acima de nove anos, com maior predisposição raças de médio e grande porte como Pastor Alemão, Golden Retriever, Akita, Terrier Escocês (Diamant et al., 1974). Nesses casos observa-se uma menor dilatação esofágica nesses pacientes quanto comparado com a forma congênita, juntamente com fraqueza de laringe, face, faringe e/ou reflexo palpebral ausente/diminuído (forma focal), ou associado a fraqueza muscular dos membros (forma generalizada (Machado et al., 2016; Stucchi et al., 2013). Para o diagnóstico definitivo dessa enfermidade, deve ser realizado a dosagem de anticorpo contra o receptor de acetilcolina, contudo, esse teste ainda não é realizado no Brasil (Dewey et al., 1997). Tendo em vista tais observações, excluiu-se esta causa no presente relato, por se tratar de um paciente filhote de pequeno porte, com uma acentuada dilatação esofágica e por apresentar reflexo palpebral normal.

Ainda, outra comorbidade descrita ao megaesôfago é a polimiosite, enfermidade que acomete com maior frequência cães adultos de grande porte, tendo como manifestações clínicas a dificuldade de deglutição, dor muscular intensa e claudicação. Seu diagnóstico é baseado no histórico do animal, sinais clínicos, e dosagem de enzimas musculares, como creatina quinase (CK) (Crivellentin \& BorinCrivelletin, 2015; Trentin et al., 2008). A dosagem de creatinina sérica da paciente encontrava-se dentro dos valores de referência, do mesmo modo que não evidenciou outras manifestações clínicas condizentes com o quadro, com exceção da disfagia.

Outras causas relatadas de megaesôfago adquirido seriam doenças endócrinas, como o hipoadrenocorticismo e hipotireoidismo, no entanto, não se observou alterações clínicas e laboratoriais (exames bioquímicos e urinálise), que poderiam presumir a presença dessas afecções. Observa-se também, megaesôfago em pacientes com hérnia de hiato, persistência do quarto arco aórtico direito, obstruções e neoplasias esofágicas, porém, os exames radiográficos não demonstraram alterações condizentes (Kook, 2013; Quessada, 1993).

O plano diagnóstico foi determinado em concordância com a literatura, que ressalta que o diagnóstico de megaesôfago congênito é realizado após a comprovação da dilatação esofágica através da anamnese, exame físico detalhado, avaliação radiográfica simples, que permite a visualização dos níveis de ar, alimentos retidos no esôfago, comorbidades secundárias à afecção e em alguns casos a dilatação, confirmado pelo esofagograma, que possibilita a observação da dilatação do órgão em toda a sua magnitude e extensão (Torres, 1997). Desta forma, as informações obtidas na anamnese e exame físico sugeriam um quadro de megaesôfago, seguido da realização de exames radiográficos simples e contrastados, o qual comprovou a suspeita. Por fim, excluiu-se causas secundárias da enfermidade, indicando então que a doença teria origem congênita.

$\mathrm{O}$ tratamento das comorbidades secundárias ao megaesôfago e adequação do manejo alimentar deve ser instituído a fim de melhorar o esvaziamento esofágico, evitando uma maior dilatação do órgão e complicações secundárias (Tanaka et al., 2010). A indicação é que o animal seja mantido, durante e alguns minutos após a alimentação, em posição bipedal pélvica sob uma plataforma elevada, da mesma maneira, deve ser fornecido alimentos pastosos fracionados em pequenas porções, várias vezes ao dia. Em circunstâncias mais graves, pode se fazer necessário a alimentação parenteral através de sondas nasogástricas. Caso haja comorbidades primárias e secundárias à afecção, o tratamento deve visar a resolução total destes quadros (Alves et al., 2013; Torres, 1997).

Visto que a canina aqui descrita não apresentava problemas secundários à doença, instituiu-se apenas o manejo alimentar enteral como método terapêutico, conforme relatado pelos autores supracitados.

Apesar da regurgitação ser um sinal clínico, Tanaka et al. (2010) recomendam a não utilização de cisaprida e metoclopramida em cães com megaesôfago idiopático, já que essa espécie não possui 
receptores para medicações procinéticas na musculatura esofágica estriada. Dessa forma, tais medicamentos não possuem efeito que justifiquem o uso, e não foram administrados no caso acompanhado.

O prognóstico para megaesôfago congênito é reservado, contudo, inúmeros pacientes apresentam melhora do quadro ao longo dos meses, caso seja realizado os cuidados intensivos com intuito de manter o aporte calórico adequado associado a correções de quaisquer enfermidades associadas (Tanaka et al., 2010). Corroborando com isso, observou-se melhora do quadro clínico da paciente até o momento do seu óbito, por causa não relacionada a doença.

\section{Conclusão}

Embora o megaesôfago seja uma enfermidade relativamente comum, nota-se uma escassez de dados em literatura que elucidem a verdadeira etiologia da forma congênita. Assim, pode-se concluir que apesar de cães de médio e grande porte apresentarem maior predisposição para o desenvolvimento do megaesôfago, raças de pequeno porte também podem ser acometidas pela doença. Além disso, frente a inexistência de tratamento curativo para a enfermidade, pode-se firmar que o manejo clínico dietético permitiu a recuperação e manutenção nutricional da paciente aqui descrita, livre de comorbidades durante o tempo de tratamento.

\section{Referências}

Alves, N. M., Dias, T. P., Pereira, A. M., Rosito, J., \& Borges, M. M. (2013). Megaesôfago congênito em cão. PUBVET, 7, 2327-2445.

Andrade, S. F., Nogueira, R. M. B., Melchert, A., Silva, M. P. C., Motta, Y. P., Brinholi, R. B., Tostes, R. A., \& Sanches, O. (2007). Megaesôfago secundário à miastenia grave em uma cadela da raça Pastor Alemão. Semina: Ciências Agrárias, 28(3), 477-481. https://doi.org/10.5433/16790359.2007v28n3p477.

Crivellentin, L. Z., \& Borin-Crivelletin, S. (2015). Casos de rotina em medicina veterinária de pequenos animais. In MedVet. MedVet.

Dewey, C. W., Bailey, C. S., Shelton, G. D., Kass, P. H., \& III, G. H. C. (1997). Clinical forms of acquired myasthenia gravis in dogs: 25 cases (1988-1995). Journal of Veterinary Internal Medicine, 11(2), 50-57. https://doi.org/10.1111/j.1939-1676.1997.tb00073.x.

Diamant, N., Szczepanski, M., \& Mui, H. (1974). Idiopathic megaesophagus in the dog: reasons for spontaneous improvement and a possible method of medical therapy. The Canadian Veterinary Journal, 15(3), 66-71.

Gelberg, H. B. (2012). Alimentary System and peritoneum, omentum, mes $\neg$ entery, and peritoneal cavity. In J. F. Zachary, D. McGavin, \& M. D. McGavin (Eds.), Pathologic Basis of Veterinary Disease (pp. 322-404). Elsevier.

Kook, P. H. (2013). Megaesophagus and other causes of esophageal dilation. Compendium on Continuing Education for the Practicing Veterinarian.

Mace, S., Shelton, G. D., \& Eddlestone, S. (2012). Megaesophagus. Compendium, 34(2), E1-E1.

Machado, L. H. A., Castro, N. C. N., Barbosa, L. C., \& Zahn, F. S. (2016). Megaesophagus secondary to myasthenia gravis. Veterinária e Zootecnia, 23(3), 347-355.

Quessada, A. M. (1993). Surgical esophageal diseases in small animals. A review. Ciência Rural, 23(2), 249-255.

Strombeck, D. R., \& Troya, L. (1976). Evaluation of lower motor neuron function in two dogs with megaesophagus. Journal of the American Veterinary Medical Association, 169(4), 411.

Stucchi, C., Gonçalves, L. A., Pelegrine, L., Alba, K. Q., Zanatta, R., Camargo, L. M., Morais, D. F., Steffens, R., Nery, C. C., \& Lemos, R. S. (2013). Evitando erros de diagnóstico em pacientes com paraparesia/plegia aguda com ênfase no botulismo. Revista de Ciências Agroveterinárias, 12(Esp.), $3-4$.

Sturgess, C. P., Canfield, P. J., Gruffydd-Jones, T. J., \& Stokes, C. R. (2001). A gross and microscopical morphometric evaluation of feline large intestinal anatomy. Journal of Comparative Pathology, 
124(4), 255-264. https://doi.org/10.1053/jcpa.2000.0460.

Tanaka, N. M., Hoogevonink, N., Tucholski, Â. P., Trapp, S. M., \& Frehse, M. S. (2010). Megaesôfago em cães. Revista Acadêmica Ciência Animal, 8(3), 271-279.

Torres, P. (1997). Megaesófago en el perro. Revisión bibliográfica y proposición de una nueva clasificación. Archivos de Medicina Veterinaria, 29(1), 13-23.

Trentin, T. de C., Campos, D. F., Dabus, D. M. M., Fazolaro, L. V., Lima, G. S., \& Pereira, R. E. P. (2008). Doenças auto-imunes em cães. Revista Cientifica Eletrônica de Medicina Veterinária, 6(10).

Histórico do artigo:

Recebido: 23 de dezembro de 2020

Aprovado: 19 de janeiro de 2021.

Disponível online: 13 de abril de 2021.
Licenciamento: Este artigo é publicado na modalidade Acesso Aberto sob a licença Creative Commons Atribuição 4.0 (CC-BY 4.0), a qual permite uso irrestrito, distribuição, reprodução em qualquer meio, desde que o autor e a fonte sejam devidamente creditados. 